\title{
A Naturalistic Study of Carbon Monoxide, Heart Rate, Oxygen Saturation, and Perfusion Index in Hookah Lounge Patrons
}

\author{
Mary P Martinasek, Allison V Calvanese, and Briana K Lipski
}

\begin{abstract}
BACKGROUND: Waterpipe (also known as hookah) smokers are exposed to several toxicants, heavy metals, and carcinogens. This study assessed physiologic data from hookah bar participants in an urban area in Florida. We hypothesized that hookah lounge patrons would have a decreased $\mathrm{S}_{\mathrm{pO}_{2}}$ and perfusion index, as well as an increased heart rate and exhaled carbon monoxide (CO) levels upon exiting the bars. METHODS: Exhaled CO levels, heart rate, pulse oximetry for carboxyhemoglobin, and perfusion index were obtained immediately before entering the hookah lounges and after exiting hookah lounges on a sample size of $\mathbf{2 0 0}$ patrons attending hookah lounges. Averages were then used for Wilcoxon signed-rank tests to assess differences across means. RESULTS: Combining data from both hookah smokers and concurrent smokers, the mean CO measurements went from $5.7 \mathrm{ppm}$ prior to lounge entry to $66.5 \mathrm{ppm}$ after the lounge visit $(P<$ .001). Conversion to carboxyhemoglobin for all hookah smokers represents an increase from $1.5 \%$ to $10.6 \%(P<.001)$. Carboxyhemoglobin mean values as measured with pulse oximetry increased from $1.4 \%$ prior to the lounge visit to $7.2 \%$ after the lounge visit $(P<.001)$. The mean heart rate for the subjects also increased from 90.3 beats/min to 94.2 beats $/$ min $(P<.001) . \mathrm{S}_{\mathrm{pO}_{2}}$ levels decreased from $98.4 \%$ to $97.8 \%$ ( $P<.001$ ). Similarly, the mean perfusion index decreased from $3.1 \%$ to $2.7 \%(P=.002)$. CONCLUSIONS: Hookah lounge smokers in the United States are exposed to high levels of $\mathrm{CO}$ that have the potential for detrimental health effects, as evidenced by rapid negative changes in physiological parameters. Respiratory therapists should be aware of potential cardiovascular changes in hookah lounge patrons who enter emergency rooms and health facilities due to recent exposures. Key words: hookah; shisha; carbon monoxide; oxygen saturation; cardiorespiratory; perfusion. [Respir Care 2021;66(2):269-274. (C) 2021 Daedalus Enterprises]
\end{abstract}

\section{Introduction}

Hookah smoking (also known as shisha, narghile, and waterpipe) is a common tradition that is believed to have begun in India and has become infused into college culture and social locations across the globe. ${ }^{1}$ Flavored tobacco (maassel) manufactured with artificial sweeteners is heated

The authors are affiliated with the Department of Health Sciences and Human Performance, University of Tampa, Tampa, Florida.

Ms Calvanese presented a version of this paper at the American Public Health Association meeting in New Orleans in 2014.

The authors have disclosed no conflicts of interest.

Correspondence: Mary P Martinasek PhD RRT, Department of Health Sciences and Human Performance, University of Tampa, 401 W Kennedy Blvd, Tampa, FL 33606. E-mail: mmartinasek@ut.edu.

DOI: $10.4187 /$ respcare. 08056 in a bowl beneath smoldering charcoal, separated by an aluminum shield or foil. Prevalence rates of hookah smoking in 2018 reported by Monitoring the Future indicated that $7.8 \%$ of high school students and $12.3 \%$ of young adults (19-30 y old) had smoked hookah in the past year. ${ }^{2}$ Although the rates have declined slightly in recent years, the data still represent 1 in 13 high schoolers and 1 in 8 young adults smoking hookah.

The prevalence of hookah smoking continues due to young adults utilizing it as means of social interaction, where groups of individuals can gather at residences or hookah lounges and pass a shared hose for smoking. ${ }^{3-5}$ The social nature, flavorings, relaxation, and low cost associated with hookah smoking makes this practice very appealing to young adults. ${ }^{5-7}$ Misperceptions of harm are found in this age group, with many young adults believing that hookah is less harmful than traditional cigarette smoking and that it doesn't contain addictive chemicals. ${ }^{7,8}$ In fact, nearly 300 chemicals have been identified in hookah smoke. ${ }^{9}$ This 


\section{Physiologic Impact of HoOKah SMOKING}

misperception may lead to young adults trying hookah when they may not have otherwise.

Hookah smoking produces toxins, heavy metals, and carcinogens that are of health concern. Smoking hookah for a typical session of 45-125 min requires the replacement of charcoal several times to sustain the strength of the smoke. The charcoal is found to produce high levels of carbon monoxide (CO). ${ }^{10-12}$ Many of the toxicants produced, including $\mathrm{CO}$, are released directly from the combustion of the charcoal in the waterpipe device. There are health concerns regarding the exposure to $\mathrm{CO}$, toxicants, polycyclic aromatic hydrocarbons, volatile organic compounds, and heavy metals. ${ }^{13-16}$ Additionally, hookah contains the addictive substance nicotine which negatively impacts the developing brain. ${ }^{17,18} \mathrm{CO}$, in particular, has lasting effects due to its strong affinity for hemoglobin (ie, > 200 times greater than that of the affinity for oxygen). ${ }^{19}$ This affinity reduces oxygen-carrying capacity in the blood and can lead to hypoxemia and cardiorespiratory disease.

Studies have been conducted in controlled settings and as quasi-experimental research to assess heart rate, blood pressure, perfusion, vascular resistance, and effects on the myocardium before and after hookah smoking. ${ }^{10,20-25}$ The majority of these studies reported increases in heart rate and $\mathrm{CO}$ after smoking hookah. One controlled study reported increases in vascular resistance both in the myocardium and the skin. ${ }^{10}$ There are limited studies in naturalistic settings, and only one other study that assessed beforeand-after measurements specifically at hookah lounges. ${ }^{26,27}$

Our study involved a naturalistic assessment of patrons attending hookah lounges. Prior to entry and upon exit from the lounges, the following data were collected: exhaled $\mathrm{CO}$ levels, carboxyhemoglobin, heart rate, $\mathrm{S}_{\mathrm{pO}_{2}}$, and perfusion index. We hypothesized that values for $\mathrm{CO}$ and heart rate would increase and that values for $\mathrm{S}_{\mathrm{pO}_{2}}$ and perfusion index would decrease in the measurements after the visit to the hookah lounge.

\section{Methods}

A total of 200 hookah smokers were recruited into this study at 6 hookah lounges in Tampa, Florida, from January 7 to February 12, 2014. The sample size was a result of the time spent in the community collecting data. Inclusion criteria consisted of anyone who went into the hookah lounges to smoke hookah and included individuals who were current cigarette smokers. After signing the consent form to participate in data collection, subjects completed a questionnaire on their demographics including age, gender, ethnicity, cigarette smoking status, and the last time they used hookah or cigarettes. Additional questions were asked after the subjects exited the hookah lounge to record the number of hookahs that were smoked and the number of active smokers. Other questions included the number of tobacco

\section{QUICK LOOK}

\section{Current knowledge}

Young adults view hookah smoking as a social activity, and this attitude is used to dismiss associated negative health effects. Acute effects include carbon monoxide toxicity, cardiovascular strain, and increased risk of infectious disease transmission. Chronic effects include COPD, cancers, and oral issues.

\section{What this paper contributes to our knowledge}

The effects of attending hookah lounges were associated with increased levels of carbon monoxide and heart rate in patrons in visits as short as $2 \mathrm{~h}$. Other changes included a decrease in perfusion and $\mathrm{S}_{\mathrm{pO}_{2}}$.

bowls smoked for each hookah, the number of charcoals used, and whether they had shared the mouthpiece with others. The questionnaire was exploratory in nature, rather than a validated instrument. Four field study researchers were trained in using a Micro $^{+}$Smokerlyzer (Bedfont Scientific, Maidstone, United Kingdom) to monitor exhaled CO and a Masimo SET Rad-57 pulse CO-oximeter (Masimo, Irvine, California). The Micro ${ }^{+}$Smokerlyzer devices were calibrated per manufacturer recommendations prior to use outside the hookah lounges.

Researchers stood outside the hookah lounges and asked entering patrons if they would participate in a voluntary research study assessing physiologic measurements and a questionnaire related to hookah smoking. The researchers stood in parking lots and on the sidewalks to avoid trespassing. If patrons voluntarily agreed to participate, then the researchers obtained their measurements and asked them to return after attending the hookah lounge for their follow-up measurements. The participants were told they would receive an incentive of a $\$ 20$ gift card upon exiting for providing follow-up data. Heart rate, $\mathrm{S}_{\mathrm{PO}_{2}}$, exhaled $\mathrm{CO}$, and pulse oximetry were obtained twice before patrons entered the hookah lounge and twice after leaving the hookah lounge. Additionally, a demographic and behavioral information survey was obtained upon exiting to obtain a better understanding of factors associated with hookah smoking.

The Micro ${ }^{+}$Smokerlyzer measured the exhaled CO in ppm and calculated the carboxyhemoglobin levels in the blood based on the exhaled measurement. Subjects were advised to take a deep breath and to hold their breath for $15 \mathrm{~s}$. At the end of the $15 \mathrm{~s}$, they exhaled slowly into the device for recorded measurements. This was repeated twice before the subjects entered the hookah lounge and twice after they had terminated smoking for reliability. The Masimo SET Rad-57 pulse CO-oximeter was used by placing the subjects' same finger in the probe to read 


\section{Physiologic Impact of HoOKah SMOKING}

various measurements: heart rate in beats/min, carboxyhemoglobin, $\mathrm{S}_{\mathrm{pO}_{2}}$, and perfusion index. Measurements were recorded once the machines stabilized at 1-2 min. There were 2 measurements recorded for reliability, and the average of the 2 measurements were used in the data analysis. All measurements were taken with the individuals in a standing position. This study was approved by the University of Tampa institutional review board.

\section{Data Analysis}

Data were analyzed using SPSS Statistics 25.0 (IBM Armonk, New York). Descriptive analyses were conducted and assumptions of statistical tests were confirmed prior to inferential data analysis. Because most of the data were not normally distributed, the Wilcoxon signed-rank test was performed for pre- and posttest measurements with the threshold for statistical significance set at $P<.05$.

\section{Results}

There was an equal distribution of male and female subjects with 104 (52\%) female hookah smokers and 96 (48\%) male hookah smokers. Subjects were between the ages of 18 and $35 \mathrm{y}$ with a mean $\pm \mathrm{SD}$ of $21.0 \pm 2.9 \mathrm{y}$. The majority of subjects $(117,58.5 \%)$ were White, 39 (19.5\%) were Hispanic/Latino, $20(10 \%)$ patrons were AfricanAmerican, 9 (4.5\%) patrons were Middle Eastern, 10 (5\%) were Asian, and $5(2.5 \%)$ self-reported being multiethnic (Table 1).

Fifty-seven $(28.5 \%)$ of the subjects were current cigarette smokers, having smoked in the past $30 \mathrm{~d}$, and the remaining $143(71.5 \%)$ subjects only smoked hookah. Of the 57 concurrent cigarette smokers, $40(15 \%)$ had smoked a cigarette in the prior $24 \mathrm{~h}$, with 30 having smoked less than a half of pack of cigarettes, $6(3 \%)$ smokers having smoked half a pack to a full pack of cigarettes, $1(0.5 \%)$ smoker having smoked a pack of cigarettes, and $1(0.5 \%)$ smoker having smoked a pack and a half of cigarettes in the prior $24 \mathrm{~h}$. The frequency that patrons reported smoking hookah was reported as following: $1(0.5 \%)$ smoker was a daily hookah smoker, $51(25.5 \%)$ smoked at least weekly but not daily, $75(37.5 \%)$ smoked at least monthly but less than weekly, $66(33 \%)$ smoked less than monthly, and 7 $(3.5 \%)$ were smoking hookah for the first time. There were $3(1.5 \%)$ subjects who reported smoking cigarettes during the hookah session; all 3 of these smokers reported smoking less than half a pack of cigarettes.

The time patrons spent inside the hookah lounge varied between 32 and 314 min with a mean \pm SD of $109.2 \pm$ $48.5 \mathrm{~min}$. The total number of charcoals and tobacco bowls smoked was divided by the number of hookah devices shared among other smokers to get the number of charcoals for each hookah. Because some hookah lounges place more
Table 1. Demographics and Smoking Characteristics of All Subjects

\begin{tabular}{lc}
\hline \hline Gender & \\
Male & $96(48)$ \\
Female & $104(52)$ \\
Ethnicity & \\
$\quad$ White & $117(58.5)$ \\
Hispanic/Latino & $39(19.5)$ \\
African-American & $20(10)$ \\
Middle Eastern & $9(4.5)$ \\
Asian & $10(5)$ \\
Multi-ethnic & $5(2.5)$ \\
Frequency of hookah use & \\
Daily & $1(0.5)$ \\
At least weekly, but not daily & $51(25.5)$ \\
At least monthly, but less than weekly & $75(37.5)$ \\
Less than monthly & $66(33)$ \\
Smoking hookah for the first time & $7(3.5)$ \\
Concurrent cigarette use* & \\
Yes & $57(28.5)$ \\
No & $143(71.5)$ \\
Time since last cigarette & \\
Within past $24 \mathrm{~h}$ & $40(20)$ \\
More than $24 \mathrm{~h}$ ago & $17(8.5)$ \\
Cigarettes smoked in past $24 \mathrm{~h}$ & \\
$<10$ & $30(15)$ \\
$<20$ & $6(3)$ \\
20 & $1(0.5)$ \\
30 & $1(0.5)$ \\
\hline Data are presented as $n$ (\%). & \\
\hline Concurrent cigarette use $=$ smoked in the past $30 \mathrm{~d}$. & \\
\hline
\end{tabular}

than one charcoal on a hookah at a time, there were more charcoals used than tobacco bowls smoked with a mean \pm SD of $2.1 \pm 1.2$ (range 1-8) charcoals and $1.5 \pm 0.7$ (range 1-4) tobacco bowls. Many subjects shared varying numbers of hookahs with different numbers of hookah smokers, the number of charcoals was multiplied by the number of hookahs used for each group and then was divided by the number of patrons who smoked together. It was estimated that patrons were each individually exposed to $1.2 \pm 0.8$ charcoals and $0.9 \pm 0.5$ tobacco bowls.

In the entire sample of subjects (hookah-only and concurrent cigarette smokers), the mean $\mathrm{CO}$ increased during the time spent in the hookah lounge from $5.7 \mathrm{ppm}$ to $66.5 \mathrm{ppm}$ $(P<.001)$, for an absolute increase of $60.9 \mathrm{ppm}$ and a relative increase of $1,068.4 \%$. The calculated measurement of carboxyhemoglobin for all hookah smokers increased from $1.5 \%$ to $10.6 \%(P<.001)$, for an absolute increase of $9.1 \%$ and a relative increase of $606.7 .0 \%$. We had measured carboxyhemoglobin with pulse oximetry, which indicated that the mean carboxyhemoglobin increased from $1.4 \%$ to $7.2 \%$ $(P<.001)$, for an absolute increase of $6.1 \%$ and a relative increase of $435.7 \%$. The mean heart rate for the subjects increased from 90.3 beats/min to 94.2 beats/min $(P<.001)$, 
Table 2. Measurements Among All Hookah Smokers

\begin{tabular}{|c|c|c|c|}
\hline \multirow{2}{*}{ Measurement } & \multicolumn{3}{|c|}{ All Hookah Smokers } \\
\hline & Before Visit & After Visit & $P$ \\
\hline Exhaled carbon monoxide, ppm & $5.7 \pm 6.3$ & $66.5 \pm 59.8$ & $<.001$ \\
\hline Carboxyhemoglobin, $\% *$ & $1.5 \pm 1.0$ & $10.6 \pm 8.1$ & $<.001$ \\
\hline Carboxyhemoglobin, $\% \dagger$ & $1.4 \pm 1.6$ & $7.2 \pm 5.3$ & $<.001$ \\
\hline Heart rate, beats/min & $90.3 \pm 14.0$ & $94.2 \pm 14.9$ & $<.001$ \\
\hline $\mathrm{S}_{\mathrm{pO}_{2}}, \%$ & $98.4 \pm 1.4$ & $97.8 \pm 1.9$ & $<.001$ \\
\hline Perfusion index & $3.1 \pm 2.4$ & $2.7 \pm 2.4$ & .002 \\
\hline
\end{tabular}

for an absolute increase of 3.8 beats/min and a relative increase of $4.2 \%$. In contrast, while $\mathrm{CO}$ and heart rate increased in hookah smokers after leaving the lounge, $\mathrm{S}_{\mathrm{pO}_{2}}$ and perfusion index decreased significantly. Mean $\mathrm{S}_{\mathrm{pO}_{2}}$ decreased from $98.4 \%$ to $97.8 \%(P<.001)$, for an absolute reduction of $-0.4 \%$ and a relative reduction of $-0.4 \%$. Similarly, the mean perfusion index decreased from $3.1 \%$ to $2.7 \%(P=.002)$, for an absolute reduction of -0.4 and a relative reduction of $-12.9 \%$ (Table 2 ).

Among the subjects who were hookah-only users, mean $\mathrm{CO}$ increased during the time spent in the hookah lounge from $3.7 \mathrm{ppm}$ to $61.6 \mathrm{ppm}(P<.001)$, for an absolute increase of $57.9 \mathrm{ppm}$ and a relative increase of 1,564.9\%. Comparatively, the calculated measurement of carboxyhemoglobin for the hookah-only smokers increased from $1.2 \%$ to $9.9 \%(P<.001)$, for an absolute increase of $8.7 \%$ and a relative increase of $725.0 \%$. The measurement of carboxyhemoglobin with pulse oximetry indicated that the mean carboxyhemoglobin values increased from $1.2 \%$ to $6.9 \%(P<.001)$, for an absolute increase of $6.0 \%$ and a relative increase of $500 \%$. The mean heart rate for hookah-only smokers increased from 88.6 beats/min to 93.7 beats/min $(P<.001)$, for an absolute increase of 5.1 beats/min and a relative increase of $5.8 \%$. Mean $\mathrm{S}_{\mathrm{pO}_{2}}$ decreased from $98.3 \%$ to $97.8 \%(P<.001)$, for an absolute reduction of $-0.5 \%$ and a relative reduction of $-0.5 \%$. The mean perfusion index decreased from $3.2 \%$ to $2.7 \%$ $(P=.02)$, for an absolute reduction of -0.3 and a relative reduction of $-9.4 \%$ (Table 3 ).

In the subset of participants who were concurrent hookah and cigarette smokers, the mean CO increased from 10.6 ppm to $78.9 \mathrm{ppm}(P<.001)$, for an absolute increase of 68.3 ppm and a relative increase of $644.3 \%$. By comparison to the calculated measurement of carboxyhemoglobin for dual users, carboxyhemoglobin increased from $2.3 \%$ to $12.5 \%$ ( $P$ $<.001$ ), for an absolute increase of $10.2 \%$ and a relative increase of $443.5 \%$. The measurement of carboxyhemoglobin with pulse oximetry indicated that the mean carboxyhemoglobin increased from $1.9 \%$ to $7.9 \%(P<.001)$, for an
Table 3. Measurements Among Hookah-Only Smokers

\begin{tabular}{lccc}
\hline \hline \multirow{2}{*}{ Measurement } & \multicolumn{3}{c}{ Hookah-Only Smokers } \\
\cline { 2 - 4 } & Before Visit & After Visit & $P$ \\
\hline Exhaled carbon monoxide, ppm & $3.7 \pm 2.7$ & $61.6 \pm 59.3$ & .001 \\
Carboxyhemoglobin, \%* & $1.2 \pm .5$ & $9.9 \pm 8.0$ & .001 \\
Carboxyhemoglobin, \%† & $1.2 \pm 1.5$ & $6.9 \pm 5.3$ & .001 \\
Heart rate, beats/min & $88.6 \pm 13.5$ & $93.7 \pm 15.2$ & .001 \\
$\mathrm{~S}_{\mathrm{pO}_{2}}, \%$ & $98.3 \pm 1.5$ & $97.8 \pm 1.3$ & .001 \\
Perfusion index & $3.2 \pm 2.4$ & $2.7 \pm 2.4$ & .02 \\
& & & \\
Data are presented as mean \pm SD. $N=143$ hookah-only smokers. \\
*Data were calculated with the Micro ${ }^{+}$Smokerlyzer CO monitor. \\
†Data were measured with a pulse oximeter. \\
\end{tabular}

Table 4. Measurement Among Concurrent Hookah and Cigarette Smokers

\begin{tabular}{lccc}
\hline \hline \multirow{2}{*}{ Measurement } & \multicolumn{3}{c}{ Concurrent Cigarette Smokers } \\
\cline { 2 - 4 } & Before Visit & After Visit & $P$ \\
\hline Exhaled carbon monoxide, ppm & $1.6 \pm 9.4$ & $78.9 \pm 59.8$ & .001 \\
Carboxyhemoglobin, \%* & $2.3 \pm 1.5$ & $12.5 \pm 8.1$ & .001 \\
Carboxyhemoglobin, \% & $1.9 \pm 1.9$ & $7.9 \pm 5.2$ & .001 \\
Heart rate, beats/min & $94.7 \pm 14.4$ & $95.4 \pm 14.2$ & .83 \\
$\mathrm{~S}_{\mathrm{pO}_{2}}, \%$ & $98.5 \pm 1.1$ & $97.9 \pm 2.9$ & .054 \\
Perfusion index & $3.0 \pm 2.5$ & $2.4 \pm 2.3$ & .03 \\
& & & \\
\hline Data are presented as mean \pm SD. $N=57$ concurrent hookah and cigarette smokers. & \\
* Data were calculated with the Micro ${ }^{+}$Smokerlyzer CO monitor. & & \\
†Data were measured with a pulse oximeter. & & & \\
\hline
\end{tabular}

absolute increase of $6.3 \%$ and a relative increase of $331.6 \%$. Although heart rate increased in dual users, the rate that it increased was not as high as that in hookah-only smokers. The mean heart rate for dual users increased from 94.7 beats/min to 95.4 beats/min $(P=.83)$, for an absolute increase of 0.7 beats $/ \mathrm{min}$ and a relative increase of $0.7 \%$. Similar to our previous findings in the entire study sample and in hookah-only users, $\mathrm{S}_{\mathrm{pO}_{2}}$ and perfusion index decreased in concurrent users as well, from $98.5 \%$ to $97.9 \%$ $(P=.054)$, for an absolute reduction of $-0.7 \%$ and a relative reduction of $-0.7 \%$. The mean perfusion index decreased from $3.0 \%$ to $2.4 \%(P=.03)$, for an absolute reduction of $-0.6 \%$ and a relative reduction of $-20 \%$ (Table 4 ).

\section{Discussion}

Our results revealed significant differences in multiple measurements in individuals after attending a hookah lounge. Our measurements included heart rate, $\mathrm{S}_{\mathrm{pO}_{2}}$, exhaled $\mathrm{CO}$, carboxyhemoglobin, and perfusion index. It is known that smoking traditional cigarettes may contribute to the increases or decreases in some of these values. Therefore, we conducted an additional data analysis and 


\section{Physiologic ImPact of HoOKah SMOKING}

removed subjects who had not smoked regular cigarettes in the past $24 \mathrm{~h}$ to account for any influence that cigarettes may have caused on the values. Comparing the literature to our results, we noted the following differences and similarities. In assessing the hookah-only smokers, heart rate increased by 5 beats/min, which was less than values found in 5 other studies, where changes ranged from 6 beats/min to as much as 22 beats/min. ${ }^{10,20,21,23,25}$ In comparing exhaled CO levels, we found a $61.6 \mathrm{ppm}$ increase in hookah-only smokers and a $68.3 \mathrm{ppm}$ increase in concurrent cigarette smokers. One other study ${ }^{23}$ noted an increase of only 8 ppm, while 4 other controlled studies reported increases in exhaled $\mathrm{CO}$ of $19-33.5$ ppm. ${ }^{10,24-26}$ We also assessed $\mathrm{S}_{\mathrm{pO}_{2}}$ with a pulse oximeter, finding slight decreases in $\mathrm{S}_{\mathrm{pO}_{2}}$, although these differences were not clinically important. We also measured perfusion index with the pulse oximeter, which is a noninvasive way to measure peripheral perfusion. Mean perfusion values dropped slightly. Two studies in the literature reported increased vascular resistance in the skin and myocardium, ${ }^{10,20}$ but we could not find any studies assessing the perfusion index.

When comparing the entire sample to just those individuals who were hookah-only smokers, we noted that cigarette smoking increased exhaled $\mathrm{CO}$ measurements, both before and after attending the hookah bars. We did not find considerable differences in the perfusion index. The heart rate variability was larger in the full sample size compared to those individuals who only smoked hookah.

Our study had several strengths and limitations. Two strengths of our study are the relatively large sample size and the capture of 2 measurements of each value both before and after the hookah lounge visit, thereby providing some reliability to the measured results. Because we asked subjects about their concurrent cigarette smoking, we were able to control for this variable in the second analysis and assess hookah-only smokers. Hookah lounges vary in size, ventilation, and population; because we captured data at 6 lounges, our data provides a good average that could be generalized to other similar areas. In our study, subjects attended hookah lounges for longer periods than in some of the controlled studies in the literature, which allowed values to reach a more stabilized plateau prior to the measurements taken after the lounge visit. Also, our heart rate measurements were obtained with a pulse oximeter, allowing time for stabilization. Unlike controlled studies in artificial settings, hookah lounges are laden with smoke, which can contribute to increased amounts of secondhand smoke. Limitations to our study include the inability to take direct measurements of carboxyhemoglobin via an arterial blood gas. In addition, subject values may also have changed because of behaviors other than hookah smoking while in the lounge that were not shared with the researchers, thereby limiting the ability to control for these variables.
Understanding the effects that hookah lounges play on these parameters is a health concern not only for individuals, but also for public safety. High levels of $\mathrm{CO}$ can affect cognitive functioning and contribute to safety concerns once a patron leaves a hookah bar and enters the roadway as a driver. Additionally, when patrons of hookah bars become ill, hospital emergency rooms should obtain a history that includes attending hookah lounges, particularly if the community is highly populated with them and the individual is a young adult. This demographic is typically drawn to social settings such as hookah lounges, especially when they are located in college campus areas. Future studies should consider gaining more accurate and complete information on the hookah charcoal that is used during a visit to the lounge. Charcoal is believed to account for much of the $\mathrm{CO}$ levels in hookah smoking, and understanding the number of charcoals may help determine effects. Patrons in our study could not recall exactly or some were uncertain of the number of charcoals utilized.

\section{Conclusions}

Hookah lounges create an environment that is conducive to derangements in physiologic functioning, leading to negative health conditions in patrons. This study provides evidence of rapid increases in $\mathrm{CO}$ levels in a very short period of time and adds to the body of knowledge on this topic. These levels put individuals at risk of side effects of $\mathrm{CO}$ toxicity. The novelty of this study includes the addition of collecting the perfusion index and overall data collection in a naturalistic setting versus a controlled setting. Future research may consider matched controls for a comparison study, which may account for variables that we were unable to measure. Actual pulse rates could be taken to ensure accuracy of heart rate and to compare to the heart rate obtained with pulse oximeters. This study provided realtime measurements in a naturalistic setting, which serves to showcase real evidence of the acute detriment caused by hookah smoking in lounges. Currently there is limited regulation on hookah lounges. This study provides evidence that can be leveraged for policy advocacy in instituting stricter requirements to protect individuals. This study provides information that respiratory therapists can utilize to educate people in the community and in classrooms for incoming practitioners. This research provides respiratory therapy students an opportunity to educate other college students on the harms of hookah smoking. Future research should consider additional research in this area to support or refute the findings.

\section{ACKNOWLEDGEMENTS}

We thank the student researchers who served as data collectors for this project, including Katelyn Bentley, Shaylin French, Dawn Howard, Kim Connor, Grace Isshiki, and Kayla Mackanin. 


\section{Physiologic Impact of HoOKah SMOKING}

\section{REFERENCES}

1. Maziak W. The waterpipe: an emerging global risk for cancer. Cancer Epidemiol 2013;37(1):1-4.

2. Johnston L, Miech R, O’Malley P, Bachman J, Schulenberg J, Patrick M. 2018 overview: key findings in adolescent drug use. Available at: https://eric.ed.gov/?id=ED578539. Accessed January 15, 2020.

3. Momenabadi V, Hossein Kaveh PhD M, Hashemi SY, Borhaninejad VR. Factors affecting hookah smoking trend in the society: a review article. Addict Health 2016;8(2):123-135.

4. Agaku I, Odani S, Armour B, Glover-Kudon R. Social aspects of hookah smoking among US youth. Pediatrics 2018;142(2):e20180341.

5. Smith-Simone S, Maziak W, Ward KD, Eissenberg T. Waterpipe tobacco smoking: knowledge, attitudes, beliefs, and behavior in two US samples. Nicotine Tob Res 2008;10(2):393-398.

6. Castañeda G, Barnett TE, Soule EK, Young ME. Hookah smoking behavior initiation in the context of millennials. Public Health 2016;137:124-130.

7. Roberts ME, Klein EG, Berman ML, Berhane B, Ferketich AK. Young adult perceptions surrounding hookah use. Health Behav Policy Rev 2017;4(6):593-600.

8. Creamer MR, Loukas A, Li X, Pasch KE, Case K, Crook B, et al. College students' perceptions and knowledge of hookah use. Drug Alcohol Depend 2016;168:191-195.

9. Shihadeh A, Schubert J, Klaiany J, El Sabban M, Luch A, Saliba NA. Toxicant content, physical properties and biological activity of waterpipe tobacco smoke and its tobacco-free alternatives. Tob Control 2015;24(Suppl 1):i22-i30.

10. Rezk-Hanna M, Nelson MD, Rader F, Benowitz NL, Rosenberry R, Chang LC, et al. Peripheral blood flow changes to cutaneous and muscular beds in response to acute hookah smoking. Am J Cardiol 2020;125(11):1725-1731.

11. Elsayed Y, Dalibalta S, Abu-Farha N. Chemical analysis and potential health risks of hookah charcoal. Sci Total Environ 2016;569-570:262268.

12. Borger J. The danger of carbon monoxide poisoning associated with hookah use: an emergency physician's perspective. N C Med J 2017;78(6):424.

13. Kassem NO, Kassem NO, Liles S, Zarth AT, Jackson SR, Daffa RM, et al. Acrolein exposure in hookah smokers and non-smokers exposed to hookah tobacco secondhand smoke: implications for regulating hookah tobacco products. Nicotine Tob Res 2018;20(4):492-501.
14. Shihadeh A, Saleh R. Polycyclic aromatic hydrocarbons, carbon monoxide, "tar", and nicotine in the mainstream smoke aerosol of the narghile water pipe. Food Chem Toxicol 2005;43(5):655-661.

15. Ashurst JV, Urquhart M, Cook MD. Carbon monoxide poisoning secondary to hookah smoking. J Am Osteopath Assoc 2012;112(10):686688.

16. El Hourani M, Talih S, Salman R, Karaoghlanian N, Karam E, El Hage R, et al. Comparison of CO, PAH, nicotine, and aldehyde emissions in waterpipe tobacco smoke generated using electrical and charcoal heating methods. Chem Res Toxicol 2019;32(6):1235-1240.

17. Torres LL, Garcia RT, Camarini R, Marcourakis T. Tobacco smoke and nicotine: neurotoxicity in brain development. In: Addictive substances and neurological disease. Amsterdam: Elsevier; 2017:273-280.

18. Dwyer JB, Broide RS, Leslie FM. Nicotine and brain development. Birth Defects Res C Embryo Today 2008;84(1):30-44.

19. Berg JM, Tymoczko JL, Stryer L. Biochemistry (loose-leaf). New York: Macmillan; 2008.

20. Nelson MD, Rezk-Hanna M, Rader F, O'Neil RM, Tang X, Shidban $\mathrm{S}$, et al. Acute effect of hookah smoking on the human coronary microcirculation. Am J Cardiol 2016;117(11):1747-1754.

21. Blank MD, Cobb CO, Kilgalen B, Austin J, Weaver MF, Shihadeh A, et al. Acute effects of waterpipe tobacco smoking: a double-blind, placebo-control study. Drug Alcohol Depend 2011;116(1-3):102-109.

22. Shaikh RB, Haque NMA, Al Mohsen HAHK, Al Mohsen AAHK, Humadi MHK, Al Mubarak ZZ, et al. Acute effects of dokha smoking on the cardiovascular and respiratory systems among UAE male university students. Asian Pac J Cancer Prev 2012;13(5):1819-1822.

23. Hakim F, Hellou E, Goldbart A, Katz R, Bentur Y, Bentur L. The acute effects of water-pipe smoking on the cardiorespiratory system. Chest 2011;139(4):775-781.

24. Jacob P, Abu Raddaha AH, Dempsey D, Havel C, Peng M, Yu L, et al. Comparison of nicotine and carcinogen exposure with water pipe and cigarette smoking. Cancer Epidemiol Biomarkers Prev 2013;22 (5):765-772.

25. Shafagoj YA, Mohammed FI. Levels of maximum end-expiratory carbon monoxide and certain cardiovascular parameters following hubble-bubble smoking. Saudi Med J 2002;23(8):953-958.

26. Kadhum M, Jaffery A, Haq A, Bacon J, Madden B. Measuring the acute cardiovascular effects of shisha smoking: a cross-sectional study. JRSM Open 2014;5(6):205427041453112.

27. Barnett TE, Curbow BA, Soule EK Jr, Tomar SL, Thombs DL. Carbon monoxide levels among patrons of hookah cafes. Am J Prev Med 2011;40(3):324-328. 